20 November 2003

Preprint-KUL-TF-2003/32

\title{
A microscopic model for Josephson currents
}

\author{
J.Lauwers, A.Verbeure \\ Instituut voor Theoretische Fysica, Katholieke Universiteit Leuven, Celestijnenlaan \\ 200D, B-3001 Leuven, Belgium \\ E-mail: joris.lauwers@fys.kuleuven.ac.be, \\ andre.verbeure@fys.kuleuven.ac.be
}

\begin{abstract}
A microscopic model of a Josephson junction between two superconducting plates is proposed and analysed. For this model, the nonequilibrium steady state of the total system is explicitly constructed and its properties are analysed. In particular, the Josephson current is rigorously computed as a function of the phase difference of the two plates and the typical properties of the Josephson current are recovered.

PACS numbers: 05.30.-d - 74.20.Fg - 03.75.Lm - 05.70.Ln
\end{abstract}

\section{Introduction}

In 1962, Josephson [1] predicted a novel phenomenon in superconductivity, namely when two different superconductors were brought into close contact. Based on elementary quantum mechanics, he predicted the existence of a supercurrent with a peculiar currentvoltage dependence. Namely, he argued that there would emerge a current of Cooper pairs which is proportional to the sine of the phase difference of the order parameters of both superconductors. The success of this prediction was immediate when indeed this phenomenon was experimentally observed already one year later [2]. It counts as one of the greatest successes of quantum mechanics in physics and you will find a chapter on the Josephson effects in almost every textbook on superconductivity. During the following decades, the increase of knowledge on this subject in theoretical solid state physics has been tremendous and applications of Josephson junctions in electronic devices have been developed [3. Progress in conceiving a microscopic theory for the Josephson effect in rigorous quantum statistical mechanics was made when Sewell obtained the Josephson and Meissner effects in an model independent approach from the assumption of offdiagonal long range order and local gauge covariance 4.

Here, we are concerned with a substantive and rigorous derivation of the Josephson current in a concrete microscopic quantum statistical model. The model consists of two twodimensional superconducting plates which have a common onedimensional contact surface through which Cooper pairs can tunnel, and as such it induces a current. We use recently developed ideas of nonequilibrium statistical mechanics to conceive a reasonable framework for this model in which the Josephson current can be computed explicitly. 
The model at hand is based on the mean field version, sometimes called the Anderson version, of the strong coupling BCS model. This model may lack many physical features, it is nevertheless an interesting model as it allows rigorous and explicit computations leading to nontrivial results.

Due to the phase difference between the superconductors, the total system is not in an equilibrium state and we have to find a reasonable description of the nonequilibrium steady state of the system. In spite of the great success of statistical mechanics for systems in equilibrium, still not very much is understood in the nonequilibrium situation, especially for quantum systems. One of the most important points in this note is thus the construction of a good nonequilibrium steady state for this model (Section 2.2). This nonequilibrium steady state is constructed by selecting the state which fulfils a list of natural conditions in this setup. This construction is then compared with other treatments found in the literature [5, 6].

After developing our framework for this model, we calculate the currents in the system (Section 3). First, we find that there is a Cooper pair current with the typical phase dependence of the traditional Josephson currents (see Figure 3). Secondly, this model can be compared with other nonequilibrium quantum statistical models for entropy production [6, 7, 8, 9] and we also compute the heat fluxes between the two superconductors. In spite of the fact that there are nontrivial particle currents in this system, we get that there are no heat fluxes or entropy production here.

\section{The microscopic model}

The model we propose consists of two superconducting plates, $I$ and $I I$. At one side, the distance between these superconductors is made very small, such that Cooper pairs can tunnel through this barrier, see Figure 1, i.e., the superconducting plates have a common contact surface.

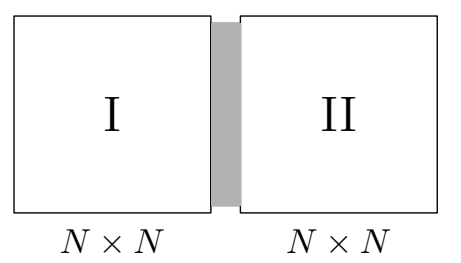

Figure 1. Two superconductors with a contact surface

We conceive a microscopic model for this setup and describe the superconductors by square lattices, i.e., $\mathbb{N}^{2} \oplus \mathbb{N}^{2}$, where the distance between the superconductors is of the order of the lattice site distance. The interactions in this system are given by local Hamiltonians $H_{N}$, i.e., for a finite lattice with $N^{2}+N^{2}$ points,

$$
H_{N}=H_{I, N}+H_{I I, N}+V_{N} \text {. }
$$

Here, $H_{I, N}$ and $H_{I I, N}$ are the Hamiltonians of the two superconductors, labelled by $I$ and $I I$, and $V_{N}$ models the interaction of the junction between the two superconductors. 
It is important to realise that the individual superconducting plates are twodimensional and that the contact is onedimensional, but nevertheless infinite in the thermodynamic limit, i.e., the limit $N \rightarrow \infty$.

The superconductors are modelled by the strong-coupling BCS model, using the quasi-spin formalism [10,11, i.e., the interactions in the superconductors are given by the Hamiltonians $H_{I, N}$ and $H_{I I, N}$ for finite $N \in \mathbb{N}$,

$H_{i, N}=\sum_{k, l=0}^{N-1} \epsilon_{I} \sigma_{i}^{z}(k, l)-\frac{1}{N^{2}} \sum_{k, l, m, n=0}^{N-1} \sigma_{i}^{+}(k, l) \sigma_{i}^{-}\left(m_{1}, n_{1}\right), \quad \epsilon_{i}>0, i=I, I I$.

These Hamiltonians $H_{i}^{N}$ act on the Hilbert space $\bigotimes_{j=0}^{(N-1)^{2}} \mathbb{C}_{j}^{2} ; \sigma_{i}^{+}, \sigma_{i}^{-}$and $\sigma_{i}^{z}=$ $\sigma_{i}^{+} \sigma_{i}^{-}-\sigma_{i}^{-} \sigma_{i}^{+}$are copies of the well-known Pauli matrices, generators of the algebra of $2 \times 2$ complex matrices $M_{2}$. The operators $\sigma_{I}^{+}(k, l)$ and $\sigma_{I}^{-}(k, l)$ represent respectively the creation and annihilation operators for a Cooper pair in superconductor $I$ with indices $(k, l)$; the $\epsilon_{i}$ are the kinetic energies of the Cooper pairs.

The junction between the two superconductors $I$ and $I I$ is modelled by

$V_{N}=-\frac{\gamma}{N} \sum_{k_{1}, k_{2}=0}^{N-1} \sigma_{I}^{+}\left(k_{1}, 0\right) \sigma_{I I}^{-}\left(k_{2}, 0\right)+\sigma_{I}^{-}\left(k_{1}, 0\right) \sigma_{I I}^{+}\left(k_{2}, 0\right), \quad \gamma \in \mathbb{R}^{+}$.

It describes the rate by which Cooper pairs tunnel through the barrier (Fig. 11). A Cooper pair with indices $\left(k_{1}, 0\right)$ in the first superconductor can tunnel through the junction and become a Cooper pair with indices $\left(k_{2}, 0\right)$ in the second superconductor. The coupling constant $\gamma$ governs the rate at which these processes occur. Note that only Cooper pairs which are on the contact surface, i.e., pairs which have a second index equal to zero, can tunnel through the junction. The BCS model (2) we use here is permutation invariant with respect to the lattice indices. The underlying geometry of the lattices plays no further role. The important point is that only $N$ out of $N^{2}$ sites of one superconductor, see equation (3), are linked with the other superconductor. The high degree of permutation symmetry in the system makes the model exactly solvable in the thermodynamic limit, $N \rightarrow \infty$.

\subsection{Equilibrium properties of the noninteracting superconductors}

Before studying the coupling between the two superconductors (3), we briefly describe the equilibrium properties and the phase transition in the noninteracting superconductors, i.e., the situation $\gamma=0$. We treat the first superconductor explicitly, the second is analogous.

Exploiting the permutation invariance of the BCS Hamiltonians (2) with respect to the lattice indices, the properties of the equilibrium states in the thermodynamic limit, $N \rightarrow \infty$, of this model can elegantly be derived using [12. The extremal $\beta_{I^{-}}$KMS or equilibrium states at inverse temperature $\beta_{I}$ in the thermodynamic limit are given by the product states $\omega_{\phi_{I}}$, where the expectation value of all $X=X_{0} \otimes X_{1} \otimes X_{2} \otimes \cdots$ in 
the infinite tensor product algebra of local observables, $\mathcal{B}_{I}=\overline{\bigcup_{B \subset \mathbb{N}^{2}} \bigotimes_{i \in B} M_{2, i}}$, is given by:

$$
\omega_{\phi_{I}}(X)=\prod_{i \in \mathbb{N}^{2}} \operatorname{tr} \rho_{\phi_{I}, i} X_{i}
$$

Here, $\rho_{\phi_{I}, i}$ is the $i$-th copy of the $2 \times 2$ density matrix $\rho_{\phi_{I}} \in M_{2}$, a solution of the selfconsistency or gap equation:

$$
\rho_{\phi_{I}}=\frac{\exp \left[-\beta_{I} h_{\phi_{I}}\right]}{\operatorname{tr} \exp \left[-\beta_{I} h_{\phi_{I}}\right]}
$$

where $h_{\phi_{I}}$ is a one-site effective or selfconsistent Hamiltonian,

$$
h_{\phi_{I}}=\epsilon_{I} \sigma_{I}^{z}-\lambda_{I} e^{i \phi_{I}} \sigma_{I}^{-}-\lambda_{I} e^{-i \phi_{I}} \sigma_{I}^{+},
$$

with an order parameter $\lambda_{I}$, satisfying the selfconsistency equation:

$$
\lambda_{I}=\left|\operatorname{tr} \rho_{\phi_{I}} \sigma_{I}^{+}\right|=\left|\omega_{\phi_{I}}\left(\sigma_{I}^{+}\right)\right| ;
$$

derived from equations (44) and (5), and with a phase $\phi_{I}$ :

$$
\phi_{I}=\arg \operatorname{tr} \rho_{\phi_{I}} \sigma_{I}^{+}=\arg \omega_{\phi_{I}}\left(\sigma_{I}^{+}\right), \quad \phi_{I} \in[0,2 \pi] .
$$

By explicit calculation, the gap equation (7) can be rewritten in the form

$$
\lambda_{I}\left(1-\frac{1}{2 k_{I}} \tanh \left[\beta_{I} k_{I}\right]\right)=0,
$$

where $k_{I}=\sqrt{\epsilon_{I}^{2}+\lambda_{I}^{2}}$ and $\left\{-k_{I}, k_{I}\right\}$ is the spectrum of the effective Hamiltonians $h_{\phi_{I}}$ (16)), and is independent of the phase $\phi_{I}$. It can readily be seen that the equation (92) has always a solution $\lambda_{I}=0$. It yields the normal phase state. Solutions $\lambda_{I} \neq 0$ exist if the following conditions are satisfied:

$$
\left\{\begin{array}{l}
\epsilon_{I}<1 / 2, \\
\beta_{I}>\beta_{c}=\frac{1}{2 \epsilon_{I}} \log \left(\frac{1+2 \epsilon_{I}}{1-2 \epsilon_{I}}\right) .
\end{array}\right.
$$

These solutions $\lambda_{I} \neq 0, \phi_{I} \in[0,2 \pi]$ are understood to describe the superconducting phase states. The order parameter $\lambda_{I} \neq 0$ (17) depends on the inverse temperature $\beta_{I}$ which has to be larger than the critical inverse temperature for the BCS model $\beta_{c}$, cf. (10). The phase of the superconductor $\phi_{I}$ (8) can be fixed freely. This leads to an infinite degeneracy of the states in the superconductive regime and is due to spontaneous symmetry breaking [13,14, 15]. The second superconductor $I I$ has analogous normal and superconducting equilibrium states.

\subsection{The nonequilibrium steady state (NESS)}

Suppose that initially the two superconductors are both in a superconducting pure phase equilibrium state at inverse temperature $\beta_{I}$ and phase $\phi_{I}$ for the first BCS superconductor and inverse temperature $\beta_{I I}$ and phase $\phi_{I I}$ for BCS superconductor II. I.e., the state of this system is the product of two pure phase states (4) , $\omega_{\phi_{I}} \otimes \omega_{\phi_{I I}}$ on the total algebra of local observables $\mathcal{B}=\overline{\bigcup_{B \subset \mathbb{N}^{2} \oplus \mathbb{N}^{2}} \bigotimes_{i \in B} M_{2, i}}$. 
If the interactions between the two superconductors are switched on, the system should ultimately evolve to a nonequilibrium steady state $\tilde{\omega}$ for the dynamics of the total interacting system (11), i.e.,

$$
\tilde{\omega}=\lim _{t \rightarrow \infty} \omega_{\phi_{I}} \otimes \omega_{\phi_{I I}} \circ \alpha_{t}
$$

where $\left\{\alpha_{t}\right\}$ is the dynamical semigroup of the total interacting infinite system, i.e.,

$$
\alpha_{t}(.)=\lim _{N \rightarrow \infty} \mathrm{e}^{\mathrm{i} t H_{N}} \cdot \mathrm{e}^{-\mathrm{i} t H_{N}} .
$$

However, it is not clear that the limit (11) exists for our model (11). Computer simulations suggest that the system continues to evolve periodically in time. Furthermore, it has to be specified in which sense the limiting dynamics $\alpha_{t}$ (12) can be defined.

To deal with these problems, Ruelle proposed the following construction for a natural nonequilibrium steady state [5, 6]:

$$
\tilde{\omega}=\lim _{T \rightarrow \infty} \frac{1}{T} \int_{0}^{T} \mathrm{~d} t \omega_{0} \circ \alpha_{t},
$$

where $\omega_{0}$ is the initial equilibrium state. Ruelle considered the case of a finite system coupled to a number of infinite reservoirs, and the conditions on the interactions between the small system and the reservoirs where such that the limiting dynamics (12) exists in norm on the $C^{*}$-algebra, i.e., independent of the state of the system. Clearly, the state $\tilde{\omega}$ (13) is an invariant state for the dynamics of the total interacting system (12) and it is called a natural nonequilibrium steady state. In recent years, exact results concerning entropy production in quantum statistical models were derived using Ruelle's framework (13), see e.g., 6, 7, 8, 9].

In our model (1), the interactions are not local and the existence and properties of the dynamics (12) depend on the state of the system. Hence, another approach has to be followed to obtain a nonequilibrium steady state. For this model, we propose to construct a steady state by selecting a good state which satisfies all the essential properties a NESS should have. Which are those properties?

First, due to the high degree in permutation symmetry in the model (11), it is natural to look for steady states in the class of product states, i.e.,

$\tilde{\omega}(X)=\prod_{i \in \mathbb{N}^{2} \oplus \mathbb{N}^{2}} \operatorname{tr} \tilde{\rho}_{i} X_{i}, \quad X=X_{k} \otimes X_{l} \otimes X_{m} \otimes \cdots \in \mathcal{B} ; X_{j} \in M_{2}$.

Furthermore, we expect the state $\tilde{\omega}$ to have the same permutation symmetries as the total Hamiltonian (11). Analysing this, we see that the total system should be divided into four parts (see Figure 2) which are permutation invariant subspaces. The first part is the bulk term of the first superconductor $\left(I_{a}\right)$, the second piece is the contact or surface part of the first superconductor $\left(I_{b}\right)$, the third part is the contact part of the second superconductor $\left(I I_{b}\right)$ and the fourth part is the bulk part of the second superconductor $\left(I I_{a}\right)$. The size of the bulk subsystems $\left(I_{a}, I I_{a}\right)$ is of the order of $O(N(N-1))$ and the subsystems on the contact surface $\left(I_{b}, I I_{b}\right)$ are of the order of $O(N)$. Hence, the states $\tilde{\omega}$ are products of four symmetric product states on these different regions:

$$
\tilde{\omega}=\tilde{\omega}_{I_{a}} \otimes \tilde{\omega}_{I_{b}} \otimes \tilde{\omega}_{I I_{b}} \otimes \tilde{\omega}_{I I_{a}} .
$$



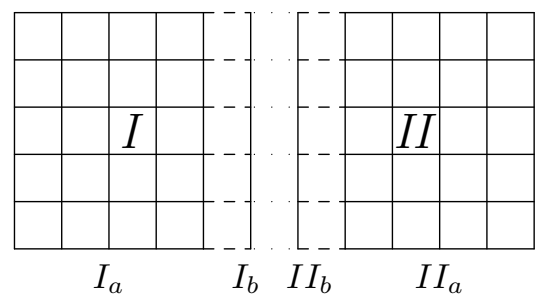

Figure 2. Devision of the system into four permutation invariant subsystems

Secondly, requiring that $\tilde{\omega}$ is a steady state is mathematically translated into the following expression:

$$
\lim _{N \rightarrow \infty} \tilde{\omega}\left(\left[H_{N}, X\right]\right)=0, \quad \forall X \in \mathcal{B} .
$$

Due to the product structure of the steady state (14), the limit $N \rightarrow \infty$ exists; it yields an equivalent Hamiltonian of the type $\tilde{H}_{N}=\sum_{i} \tilde{h}_{i}$, and the condition for the time invariance becomes now:

$$
\tilde{\omega}\left(\left[\tilde{h}_{i}, X_{i}\right]\right)=0, \quad \forall X_{i} \in M_{2, i},
$$

where $\tilde{h}_{i} \in M_{2, i}$ is the site dependent effective one-site Hamiltonian for site $i \in \mathbb{N}^{2} \oplus \mathbb{N}^{2}$. It follows directly from the permutation symmetries of $H_{N}$ (11) and the product structure of the state $\tilde{\omega}(14)$ that these effective Hamiltonians have the following structure:

$$
\tilde{h}_{i}=\left\{\begin{array}{cl}
\epsilon_{I} \sigma_{I}^{z}(i)-\tilde{\omega}\left(\sigma_{I_{a}}^{+}\right) \sigma_{I_{a}}^{-}(i)-\tilde{\omega}\left(\sigma_{I_{a}}^{-}\right) \sigma_{I_{a}}^{+}(i), & i \in I_{a} \\
\epsilon_{I} \sigma_{I_{b}}^{z}(i)-\tilde{\omega}\left(\sigma_{I_{a}}^{+}\right) \sigma_{I_{b}}^{-}(i)-\tilde{\omega}\left(\sigma_{I_{a}}^{-}\right) \sigma_{I_{b}}^{+}(i) & \\
-\gamma\left(\tilde{\omega}\left(\sigma_{I I_{b}}^{+}\right) \sigma_{I_{b}}^{-}(i)+\tilde{\omega}\left(\sigma_{I I_{b}}^{-}\right) \sigma_{I_{b}}^{+}(i)\right), & i \in I_{b} \\
\epsilon_{I I} \sigma_{I I_{b}}^{z}(i)-\tilde{\omega}\left(\sigma_{I I_{a}}^{+}\right) \sigma_{I I_{b}}^{-}(i)-\tilde{\omega}\left(\sigma_{I I_{a}}^{-}\right) \sigma_{I I_{b}}^{+}(i) & \\
-\gamma\left(\tilde{\omega}\left(\sigma_{I_{b}}^{+}\right) \sigma_{I I_{b}}^{-}(i)+\tilde{\omega}\left(\sigma_{I_{b}}^{-}\right) \sigma_{I I_{b}}^{+}(i)\right), & i \in I I_{b} \\
\epsilon_{I I} \sigma_{I I_{b}}^{z}(i)-\tilde{\omega}\left(\sigma_{I I_{a}}^{+}\right) \sigma_{I I_{b}}^{-}(i)-\tilde{\omega}\left(\sigma_{I I_{a}}^{-}\right) \sigma_{I I_{b}}^{+}(i), & i \in I I_{a}
\end{array}\right.
$$

where $\sigma_{t}^{\#}$ is the $t$-th copy of the Pauli matrix $\sigma^{\#}$ in the site $t \in\left\{I_{a}, I_{b}, I I_{a}, I I_{b}\right\}$. The expectation values $\tilde{\omega}\left(\sigma_{t}^{ \pm}\right)$appearing in these expressions are the order parameters for this effective Hamiltonian. They are determined in a selfconsistent way, see below, by the effective Hamiltonian $\tilde{h}$ (16) and the invariant state $\tilde{\omega}$ (15).

A third requirement is that a NESS $\tilde{\omega}$ should have the same properties as the initial equilibrium state $\omega_{\phi_{I}} \otimes \omega_{\phi_{I I}}$ if we look at the system far enough away from the contact surface, i.e., the expectation values for local observables $X \in \mathcal{B}$ with a support in $I_{a}$ or $I I_{a}$ satisfy:

$$
\begin{aligned}
& \tilde{\omega}(X)=\omega_{\phi_{I}}(X), \quad \text { for } X \text { with support in } I_{a} ; \\
& \tilde{\omega}(X)=\omega_{\phi_{I I}}(X), \quad \text { for } X \text { with support in } I I_{a} .
\end{aligned}
$$

In this sense, the steady state $\tilde{\omega}$ has a memory of the initial equilibrium state $\omega_{\phi_{I}} \otimes \omega_{\phi_{I I}}$, a property which seems indeed a natural ingredient for a NESS. 
The NESS can now be constructed explicitly as follows. The requirement that $\tilde{\omega}$ is an invariant product state (15) for the dynamics induced by $\tilde{h}$ (16) leads to the following choice for the one-site density matrices $\tilde{\rho}_{i}$ of $\tilde{\omega}$ (14):

$$
\tilde{\rho}_{i}=c_{+}|\tilde{+}\rangle\left\langle\left.\tilde{+}\right|_{i}+c_{-} \mid \tilde{\sim}\right\rangle\left\langle\left.\tilde{-}\right|_{i}, \quad \forall i \in \mathbb{N}^{2} \oplus \mathbb{N}^{2},\right.
$$

where $|\tilde{+}\rangle_{i}$ and $|\tilde{-}\rangle_{i}$ are the eigenvectors of $\tilde{h}_{i}$, and the constants $c_{-}$and $c_{+}$are two positive constants adding up to one. A natural way to implement condition (17) is to to define the constants $c_{-}$and $c_{+}$by means of the expectation values of the one-site density matrices of the initial equilibrium state $\omega_{\phi_{I}} \otimes \omega_{\phi_{I I}}$ on the eigenstates of $\tilde{h}_{i}$, i.e., we chose

$$
c_{+}=\left\langle\tilde{+}\left|\rho_{0, i}\right| \tilde{+}\right\rangle_{i}, \quad \text { and }, \quad c_{-}=\left\langle\tilde{-}\left|\rho_{0, i}\right| \tilde{\sim}\right\rangle_{i} .
$$

In other words, for every site $i$ in both superconductors, we project the local one-site density matrices $\rho_{0, i}$ of the initial equilibrium state $\omega_{\phi_{I}} \otimes \omega_{\phi_{I I}}$ onto the subalgebra in $M_{2}$ which is invariant under the dynamics induced by $\tilde{h}$ (16). Hence, the one-site density matrices $\tilde{\rho}_{i}$ of the NESS are given by

$$
\tilde{\rho}_{i}=|\tilde{+}\rangle\left\langle\tilde{+}\left|\left\langle\tilde{+}, \rho_{0} \tilde{+}\right\rangle_{i}+\right| \tilde{-}\right\rangle\langle\tilde{-}|\left\langle\tilde{-}, \rho_{0} \tilde{-}\right\rangle_{i}, \quad \forall i \in \mathbb{N}^{2} \oplus \mathbb{N}^{2} .
$$

Furthermore, in order to meet condition (17) and to avoid trivial nonsuperconducting solutions in (18), we explicitly set the bulk order parameters and phases equal to the equilibrium order parameters and phases, i.e.,

$\tilde{\omega}\left(\sigma_{I_{a}}^{+}\right)=\lambda_{I} \mathrm{e}^{\mathrm{i} \phi_{I}}=\omega_{\phi_{I}}\left(\sigma_{I}^{+}\right), \quad$ and,$\quad \tilde{\omega}\left(\sigma_{I I_{a}}^{+}\right)=\lambda_{I} \mathrm{e}^{\mathrm{i} \phi_{I I}}=\omega_{\phi_{I I}}\left(\sigma_{I I}^{+}\right)$.

It is easy to check that this choice (18)-(19) yields a state $\tilde{\omega}$ fulfilling indeed all of the above requirements. By construction, this state is again a product state (14) and it is an invariant state under the limiting dynamics (15). In sites which do not belong to the contact surface, i.e., $i \neq\left(k_{1}, 0\right) \in I_{b}$ and $i \neq\left(k_{2}, 0\right) \in I I_{b}$, the local density matrices of the initial equilibrium state and the constructed NESS coincide (17). This can be seen as follows. In the bulk regions, i.e., for sites $i \in I_{a} \cup I I_{a}$, the effective Hamiltonians $\tilde{h}_{i}$ (16) coincide with the equilibrium effective Hamiltonians $h_{\phi_{I}}$ or $h_{\phi_{I I}}$ (6). Hence in $I_{a} \cup I I_{a}$, the spectral decompositions of $\rho_{\phi_{I}}$ or $\rho_{\phi_{I I}}$ coincide with those given in (18).

Now, we have to compute the order parameters and phases in the effective Hamiltonians (16). Compared to the equilibrium situation, see section 2.1, where two order parameters (7) and two phases (8) completely determine the equilibrium states, we have now four different order parameters and four phases appearing, cf. (16), i.e., apart from the parameters in Equation (19), we also have to compute

$$
\begin{array}{lll}
\tilde{\lambda}_{I}=\left|\tilde{\omega}\left(\sigma_{I_{b}}^{+}\right)\right|, & \text {and, } & \tilde{\phi}_{I}=\arg \tilde{\omega}\left(\sigma_{I_{b}}^{+}\right) ; \\
\tilde{\lambda}_{I I}=\left|\tilde{\omega}\left(\sigma_{I I_{b}}^{+}\right)\right|, & \text {and }, & \tilde{\phi_{I I}}=\arg \tilde{\omega}\left(\sigma_{I I_{b}}^{+}\right),
\end{array}
$$

Using (18) and (19), we find an explicit expression for the selfconsistency equations in the steady state $\tilde{\omega}$ which determine these order parameters and phases:

$$
\tilde{\lambda}_{I} \mathrm{e}^{\mathrm{i} \tilde{\phi}_{I}}=\tilde{\omega}\left(\sigma_{I}^{+}\left(k_{1}, 0\right)\right)
$$




$$
\begin{aligned}
& =\left(\lambda_{I} \mathrm{e}^{\mathrm{i} \phi_{I}}+\gamma \tilde{\lambda}_{I I} \mathrm{e}^{\mathrm{i} \tilde{\phi}_{I I}}\right) \frac{\epsilon_{I}^{2}+\lambda_{I}\left|\lambda_{I} \mathrm{e}^{\mathrm{i} \phi_{I}}+\gamma \tilde{\lambda}_{I I} \mathrm{e}^{\mathrm{i} \tilde{\phi}_{I I}}\right| \cos \left(\phi_{I}-\tilde{\phi}_{I}\right)}{\epsilon_{I}^{2}+\left|\lambda_{I} \mathrm{e}^{\mathrm{i} \phi_{I}}+\gamma \tilde{\lambda}_{I I} \mathrm{e}^{\mathrm{i} \tilde{\phi}_{I I}}\right|^{2}} \\
\tilde{\lambda}_{I I} \mathrm{e}^{\mathrm{i} \tilde{\phi}_{I I}} & =\tilde{\omega}\left(\sigma_{I I}^{+}\left(k_{2}, 0\right)\right) \\
& =\left(\lambda_{I I} \mathrm{e}^{\mathrm{i} \phi_{I I}}+\gamma \tilde{\lambda}_{I} \mathrm{e}^{\mathrm{i} \tilde{\phi}_{I}}\right) \frac{\epsilon_{I I}^{2}+\lambda_{I I}\left|\lambda_{I I} \mathrm{e}^{\mathrm{i} \phi_{I I}}+\gamma \tilde{\lambda}_{I} \mathrm{e}^{\mathrm{i} \tilde{\phi}_{I}}\right| \cos \left(\phi_{I I}-\tilde{\phi}_{I I}\right)}{\epsilon_{I I}^{2}+\left|\lambda_{I I} \mathrm{e}^{\mathrm{i} \phi_{I I}}+\gamma \tilde{\lambda}_{I} \mathrm{e}^{\mathrm{i} \tilde{\phi}_{I}}\right|^{2}}
\end{aligned}
$$

Together with the selfconsistency equations for $\lambda_{I}$ and $\lambda_{I I}$ (9), the above equations form a set of six coupled transcendental equations whose solutions completely determine the state $\tilde{\omega}(18)$.

The steady state $\tilde{\omega}(18)$ divides the system into four parts. In both superconductors away from the contact surface, see Fig. 2, the presence of the other superconductor is not felt. The system is unperturbed and behaves as in the initial equilibrium state. Nevertheless, the NESS (18) is not normal with respect to the equilibrium state $\omega_{\phi_{I}} \otimes \omega_{\phi_{I I}}$, because the one-site density matrices $\tilde{\rho}_{i}$ on an infinite amount of sites on the contact surface (i.e., the sites $i \in I_{b} \cup I I_{b}$ ) differ from the one-site density matrices of the equilibrium state (5). On the contact surface, the system is effectively disturbed and there appear two intermediate layers with different properties compared with the initial equilibrium state.

Finally, let us compare this construction of a NESS with the construction proposed by Ruelle (13). The essential relation between our construction (18) and Ruelle's construction (13) is that in the latter one, it can be expected that the parts of the algebra which are not invariant under the dynamics (12) are averaged out and that the NESS is effectively a projection of the initial state on the subalgebra which is invariant under the dynamics, i.e., it leads to the same state as in (18).

This finishes the construction of our framework. Let us summarise the situation at hand. We start with the microscopic model (11) of two different superconductors interacting via a particular Josephson junction and we constructed a particular reasonable nonequilibrium steady state $\tilde{\omega}$ with site local density matrices given by Equations (18)-(21).

\section{Transport phenomena}

In the framework described above, we can investigate the nonequilibrium properties and the existence of currents which emerge by bringing two superconductors in contact with each other. In particular, we compute the expression for the Josephson particle current.

Suppose $Q_{N}$ is an extensive observable, e.g., the total number of particles, the energy,... The transport related to this quantity $j(Q)$ in the steady state (18) is defined as,

$$
j(Q)=\lim _{N \rightarrow \infty} \mathrm{i} \frac{1}{N} \tilde{\omega}\left(\left[H_{N}, Q_{N}\right]\right)
$$

where $H_{N}$ is the Hamiltonian of the total interacting system (11). In this definition, the current is scaled with the size of the contact surface, i.e., we divide by $N$. This is the 
right scaling to obtain density quantities. Therefore, $j(Q)$ is the current density. Taking into account the product character of the steady state (18) and the fact that this state is invariant only under the limiting dynamics (15), we compute

$$
\begin{aligned}
\mathrm{i} \frac{1}{N} \tilde{\omega}\left(\left[H_{N}, Q_{N}\right]\right) & =\mathrm{i} \tilde{\omega}\left(\left[\sum_{k_{1}, l_{1}=0}^{N-1} \epsilon_{I} \sigma_{I}^{z}\left(k_{1}, l_{1}\right)+\sum_{k_{2}, l_{2}=0}^{N-1} \epsilon_{I I} \sigma_{I I}^{z}\left(k_{2}, l_{2}\right)\right.\right. \\
& -\frac{N(N-1)}{N^{2}} \sum_{k_{1}, l_{1}=0}^{N-1} \sigma_{I}^{+}\left(k_{1}, l_{1}\right) \lambda_{I} \mathrm{e}^{-\mathrm{i} \phi_{I}}+\sigma_{I}^{-}\left(k_{1}, l_{1}\right) \lambda_{I} \mathrm{e}^{\mathrm{i} \phi_{I}} \\
& -\frac{1}{N} \sum_{k_{1}, l_{1}=0}^{N-1} \sigma_{I}^{+}\left(k_{1}, l_{1}\right) \tilde{\lambda}_{I} \mathrm{e}^{-\mathrm{i} \tilde{\phi}_{I}}+\sigma_{I}^{-}\left(k_{1}, l_{1}\right) \tilde{\lambda}_{I} \mathrm{e}^{\mathrm{i} \tilde{\phi}_{I}} \\
& -\gamma \sum_{k_{1}=0}^{N-1} \sigma_{I}^{+}\left(k_{1}, 0\right) \tilde{\lambda}_{I I} \mathrm{e}^{-\mathrm{i} \tilde{\phi}_{I I}}+\sigma_{I}^{-}\left(k_{1}, 0\right) \tilde{\lambda}_{I I} \mathrm{e}^{\mathrm{i} \tilde{\phi}_{I I}} \\
& -\frac{N(N-1)}{N^{2}} \sum_{k_{2}, l_{2}=0}^{N-1} \sigma_{I I}^{+}\left(k_{2}, l_{2}\right) \lambda_{I I} \mathrm{e}^{-\mathrm{i} \phi_{I I}}+\sigma_{I}^{-}\left(k_{2}, l_{2}\right) \lambda_{I I} \mathrm{e}^{\mathrm{i} \phi_{I I}} \\
& -\frac{1}{N} \sum_{k_{2}, l_{2}=0}^{N-1} \sigma_{I I}^{+}\left(k_{2}, l_{2}\right) \tilde{\lambda}_{I I} \mathrm{e}^{-\mathrm{i} \tilde{\phi}_{I I}}+\sigma_{I I}^{-}\left(k_{2}, l_{2}\right) \tilde{\lambda}_{I I} \mathrm{e}^{\mathrm{i} \tilde{\phi}_{I I}} \\
& \left.\left.-\gamma \sum_{k_{2}=0}^{N-1} \sigma_{I I}^{+}\left(k_{2}, 0\right) \tilde{\lambda}_{I} \mathrm{e}^{-\mathrm{i} \tilde{\phi}_{I}}+\sigma_{I I}^{-}\left(k_{2}, 0\right) \tilde{\lambda}_{I} \mathrm{e}^{\mathrm{i} \tilde{\phi}_{I}}, Q_{N}\right]\right) .
\end{aligned}
$$

This is simplified using that $\tilde{\omega}$ is invariant under the limiting dynamics, i.e., $\tilde{\omega}\left(\left[\sum_{i} \tilde{h}_{i}, Q_{N}\right]\right)=0$ (15). We obtain

$\mathrm{i} \frac{1}{N} \tilde{\omega}\left(\left[H_{N}, Q_{N}\right]\right)=\mathrm{i} \frac{1}{N^{2}} \tilde{\omega}\left(\left[-\sum_{k_{1}, l_{1}=0}^{N-1} X_{I}\left(k_{1}, l_{1}\right)-\sum_{k_{2}, l_{2}=0}^{N-1} X_{I I}\left(k_{2}, l_{2}\right), Q_{N}\right]\right)$,

where

$X_{I}\left(k_{1}, l_{1}\right)=\sigma_{I}^{+}\left(k_{1}, l_{1}\right)\left(\tilde{\lambda}_{I} \mathrm{e}^{\mathrm{i} \tilde{\phi}_{I}}-\lambda_{I} \mathrm{e}^{\mathrm{i} \phi_{I}}\right)+\sigma_{I}^{-}\left(k_{1}, l_{1}\right)\left(\tilde{\lambda}_{I} \mathrm{e}^{-\mathrm{i} \tilde{\phi}_{I}}-\lambda_{I} \mathrm{e}^{-\mathrm{i} \phi_{I}}\right)$,

and

$X_{I I}\left(k_{2}, l_{2}\right)=\sigma_{I I}^{+}\left(k_{2}, l_{2}\right)\left(\tilde{\lambda}_{I I} \mathrm{e}^{\mathrm{i} \tilde{\phi}_{I I}}-\lambda_{I I} \mathrm{e}^{\mathrm{i} \phi_{I I}}\right)+\sigma_{I I}^{-}\left(k_{2}, l_{2}\right)\left(\tilde{\lambda}_{I I} \mathrm{e}^{-\mathrm{i} \tilde{\phi}_{I I}}-\lambda_{I I} \mathrm{e}^{-\mathrm{i} \phi_{I I}}\right)$.

The order parameters and phases, $\lambda_{I}, \tilde{\lambda}_{I}, \ldots$, appearing in these expressions are defined in Equations (91), (201) and (21). Already from this expression (23), it is clear that the general current density $j(Q)$ for arbitrary $Q$ (see Eq. (22)) is of order $O(1)$. In the following sections, we compute the transport of two important quantities.

\subsection{Particle current}

Of course, the most interesting transport phenomenon in this model is the Cooper pair current through the junction. Is their really a current and what are its properties? The 


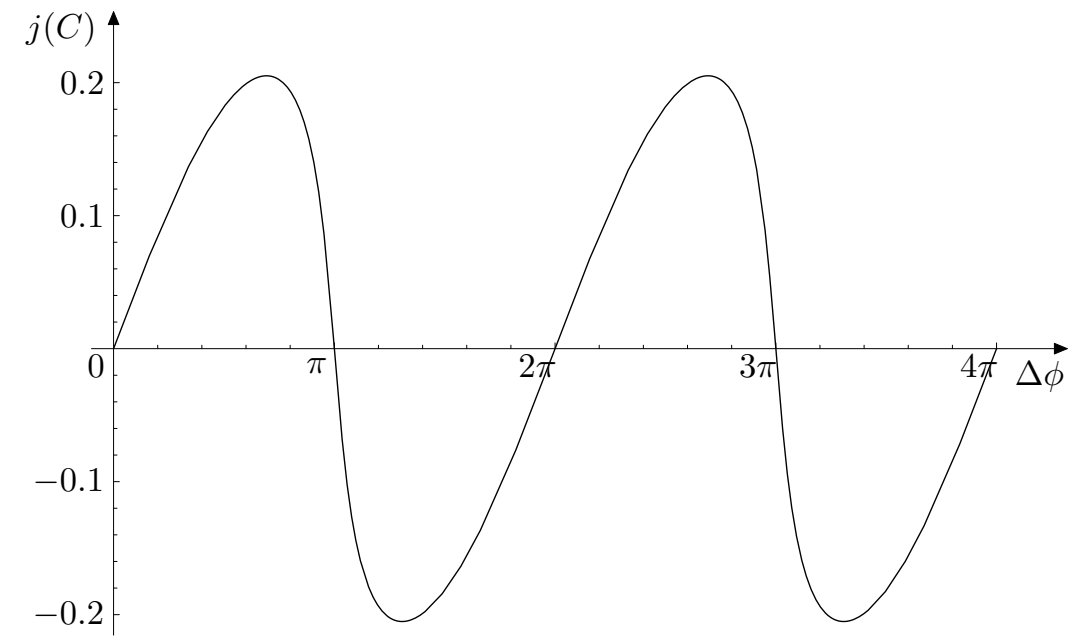

Figure 3. Dependence of the Josephson current $j(C)$ on the phase difference $\Delta \phi$

relative particle current is defined as the transport of the relative number operator $C_{N}$

$$
C_{N}=\sum_{k_{1}, l_{1}=0}^{N-1} \sigma_{I}^{+}\left(k_{1}, l_{1}\right) \sigma_{I}^{-}\left(k_{1}, l_{1}\right)-\sum_{k_{2}, l_{2}=0}^{N-1} \sigma_{I I}^{+}\left(k_{2}, l_{2}\right) \sigma_{I I}^{-}\left(k_{2}, l_{2}\right)
$$

Calculating $j(C)$ using equation (23), we find

$$
j(C)=2\left(\lambda_{I} \tilde{\lambda}_{I} \sin \left(\phi_{I}-\tilde{\phi}_{I}\right)-\lambda_{I I} \tilde{\lambda}_{I I} \sin \left(\phi_{I I}-\tilde{\phi}_{I I}\right)\right)
$$

The order of magnitude of the total flux of Cooper pairs is proportional to the size of the contact surface. It depends explicitly on the sines of the phase differences between the bulk and the contact surface, i.e., $\phi_{i}-\tilde{\phi}_{i}$ in both superconductors, $i=I, I I$, and on the order parameters, see (19)-(21). However, the only free parameters are the bulk phases $\phi_{I}$ and $\phi_{I I}$, the other parameters are determined by the selfconsistency equations (201)(21). Hence, the interesting point here is the dependence of the Cooper pair current on the phase difference between the two (bulk) superconductors $\Delta \phi=\phi_{I I}-\phi_{I}$. Let us illustrate this with an example. In Figure 3, we plotted the Josephson current $j(C)$ (24) as a function of the phase difference $\Delta \phi=\phi_{I I}-\phi_{I}$. For this figure, we took $\beta_{I}=\beta_{I I}=4, \epsilon_{I}=\epsilon_{I I}=1 / 4$ and $\gamma=1 / 2$. If the phase difference is zero $\Delta \phi=0$, there is no Cooper pair current in the system, and if the sign of the phase difference is inverted, also the direction of the superconducting current is reversed. Hence, this supercurrent $j(C)$ (24) has the basic properties of the superconducting tunnel current predicted by Josephson [1. In the textbook derivations of the Josephson current, the current is proportional to the sine of the phase difference $\Delta \phi$. In this model, however, the relation with the phase difference is more complex (24), but as can be seen in the example (Figure 31), it has a similar sinusoidal dependence on the phase difference of the order parameters in both superconductors. 


\subsection{Heat fluxes and entropy production}

Here, we want to make a link with the current research about entropy production in quantum statistical models 6,7]16]. We investigate the entropy production in our model and compare different notions.

In quantum systems when the time evolution is given by a unitary conservative dynamics, it is known that the von Neumann entropy does not change. This unitary time evolution however, induces correlations between different parts of our large system. It is natural to think of an observer who can only observe part of the large system. When after some time the correlations of this part with other parts in the large system are forgotten, the entropy of this subsystem has changed, or entropy has been produced. In this way, entropy production can be understood as the change of entropy when partitioning the total system in different parts [16].

Applying this idea to our model and considering the entropy production induced by the partitioning of the total system into the two superconductors, we find the following expression for the entropy production [16]:

$$
e_{1}=\lim _{N \rightarrow \infty}-\mathrm{i} \frac{1}{N} \tilde{\omega}\left(\left[H_{N},\left.\log \tilde{\omega}\right|_{I}+\left.\log \tilde{\omega}\right|_{I I}\right]\right),
$$

where $\left.\tilde{\omega}\right|_{I}$ and $\left.\tilde{\omega}\right|_{I I}$ are the density matrices of the state $\tilde{\omega}$ restricted to resp. the first and the second superconductor.

However, due to the product character of the steady state and using cyclic permutations under the trace, it is straightforward to check that the expectation value of this commutator vanishes. Hence, there is no entropy production in this model, i.e., $e_{1}=0$.

The definition (25) can be compared with the conventional notion of entropy production, e.g., see [6,7], where the entropy production is defined as the sum of the heat fluxes in the system, i.e., we calculate the current (22) associated with the following observable

$$
Q_{N}=\beta_{I} H_{I, N}+\beta_{I I} H_{I I, N},
$$

where $\beta_{I}$ and $\beta_{I I}$ are the initial inverse temperatures of the equilibrium states of the two superconductors and $H_{I, N}$ and $H_{I I, N}$ are the BCS Hamiltonians (2). They represent the internal energy in the superconductors. This leads to the following current (22):

$$
\begin{aligned}
e_{2}=j(Q)= & \lim _{N \rightarrow \infty} \frac{2}{N} \beta_{I}\left(\tilde{\omega}\left(\sigma_{I}^{z}\left(k_{1}, 0\right)\right)+2 \epsilon_{I}\right) \lambda_{I} \tilde{\lambda}_{I} \sin \left(\phi_{I}-\tilde{\phi}_{I}\right) \\
& +\frac{2}{N} \beta_{I I}\left(\tilde{\omega}\left(\sigma_{I I}^{z}\left(k_{2}, 0\right)\right)+2 \epsilon_{I I}\right) \lambda_{I I} \tilde{\lambda}_{I I} \sin \left(\phi_{I I}-\tilde{\phi}_{I I}\right) \\
= & 0 .
\end{aligned}
$$

Hence, the first definition (25) of entropy production and the conventional definition (22) in terms of heat fluxes both lead to zero entropy production. However, for the last case, we see that the heat fluctuations are of order $O\left(N^{-1}\right)$ around zero. Also, there is no definite sign for the entropy production fluctuations (26) in this state as the sign depends on the phase differences, i.e., just as in the case of the Josephson supercurrent 
(24), but these heat currents are of a lower order of magnitude. This way, we may conclude that there are no heat fluxes in this system, there are only small fluctuations around zero.

Finally, remark that in our setup, we took twodimensional superconductors and a onedimensional contact surface. Of course, it might be interesting to look at other dimensions for the superconductors and the contact surface. We expect that our methods and results remain valid in those situations. The onedimensional case, however, is basically different. If the contact surface is finite in the thermodynamic limit, the nonequilibrium steady state does not factorise in the contact region.

\section{Acknowledgements}

We wish to thank Christian Maes and Wojciech De Roeck for useful discussions. J. Lauwers gratefully acknowledges het Bijzonder Onderzoeksfonds K.U.Leuven for financial support.

\section{References}

[1] B. D. Josephson. Possible new effects in superconductive tunnelling. Phys. Lett., 1:251 - 253, 1962.

[2] P. W. Anderson and J. M. Rowell. Probable observation of the josephson superconducting tunneling effect. Phys. Rev. Lett., 10:230 - 232, 1963.

[3] K. K. Likharev. Dynamics of Josephson junctions and circuits. Gordon and Breach, New York, 1986.

[4] G. L. Sewell. Off-diagonal long range order and superconductive electrodynamics. J. Math. Phys., $38: 2053-2071,1997$.

[5] D. Ruelle. Natural nonequilibrium states in quantum statistical mechanics. J. Stat. Phys., 98:57 $-75,2000$.

[6] D. Ruelle. Entropy production in quantum spin systems. Commun. Math. Phys., 224:3 - 16, 2001.

[7] V. Jakšić and C. A. Pillet. Mathematical theory of non-equilibrium quantum statistical mechanics. J. Stat. Phys., 108:787 - 829, 2002.

[8] W. H. Aschbacher and C. A. Pillet. Non-equilibrium steady states of the XY chain. J. Stat. Phys., 112:1153 - 1175, 2003.

[9] T. Matsui and Y. Ogata. Variational principle for non-equilibrium steady states of the XX model. to appear in Rev. Math. Phys., 16:?? - ??, 2003. mp-arc/03-34.

[10] W. Thirring and A. Wehrl. On the mathematical structure of the B.C.S.-model. Commun. Math. Phys., 4:303 - 314, 1967.

[11] W. Thirring. On the mathematical structure of the B.C.S.-model II. Commun. Math. Phys., $7: 181-189,1968$.

[12] M. Fannes, H. Spohn, and A. Verbeure. Equilibrium states for mean-field models. J. Math. Phys., 21:355-358, 1980.

[13] D. Goderis, A. Verbeure, and P. Vets. Fluctuation oscillations and symmetry breaking: the BCSmodel. Il Nuovo Cimento, 106 B:375 - 383, 1991.

[14] T. Michoel and A. Verbeure. Goldstone boson normal coordinates. Commun. Math. Phys., 216:461 $-490,2001$.

[15] J. Lauwers and A. Verbeure. Metastability in the BCS-model. J. Phys. A: Math. Gen., 34:5517 $-5530,2001$. 
[16] D. Ruelle. How should one define entropy production for nonequilibrium quantum spin systems? Rev. Math. Phys., 14:701-707, 2002. 